\title{
Short-Term Repeated-Sprint Training in Hot and Cool Conditions Similarly Benefits Performance in Team-Sport
} Athletes

\author{
Julien D. Périard ${ }^{1 *}$, David B. Pyne ${ }^{1}$, David J. Bishop ${ }^{2}$, Alice Wallett ${ }^{1}$ and Olivier Girard ${ }^{3}$ \\ ${ }^{1}$ Research Institute for Sport and Exercise, University of Canberra, Canberra, ACT, Australia, ${ }^{2}$ Institute of Health and Sport, \\ Victoria University, Melbourne, VIC, Australia, ${ }^{3}$ School of Human Sciences (Exercise and Sport Science), The University of \\ Western Australia, Perth, WA, Australia
}

OPEN ACCESS

Edited by:

James (Jim) David Cotter,

University of Otago, New Zealand

Reviewed by:

Daniel Gagnon,

Université de Montréal, Canada

Jessica Anne Mee,

University of Worcester,

United Kingdom

Michael John Zurawlew,

Liverpool John Moores University,

United Kingdom

*Correspondence:

Julien D. Périard

julien.periard@canberra.edu.au

Specialty section:

This article was submitted to

Exercise Physiology,

a section of the journal

Frontiers in Physiology

Received: 10 June 2020

Accepted: 27 July 2020

Published: 27 August 2020

Citation:

Périard JD, Pyne DB, Bishop DJ,

Wallett $A$ and Girard O (2020)

Short-Term Repeated-Sprint Training in Hot and Cool Conditions Similarly

Benefits Performance in

Team-Sport Athletes.

Front. Physiol. 11:1023.

doi: 10.3389/fphys.2020.01023
This study compared the performance and physiological adaptations of short-term repeated-sprint training in $\mathrm{HOT}\left[40^{\circ} \mathrm{C}\right.$ and $40 \%$ relative humidity $\left.(\mathrm{RH})\right]$ and $\mathrm{COOL}\left(20^{\circ} \mathrm{C}\right.$ and $40 \% \mathrm{RH}$ ) conditions in team-sport athletes. Twenty-five trained males completed five training sessions of 60 min over 7 days in HOT $(n=13)$ or COOL $(n=12)$ conditions, consisting of a submaximal warm-up and four sets of maximal sprints. Before and after the intervention, intermittent shuttle running performance was assessed in cool and repeated-sprint ability in hot conditions; the latter preceded and followed by neuromuscular function testing. During the repeated-sprint training sessions, skin $\left(\sim 8.4^{\circ} \mathrm{C}\right)$ and core $\left(\sim 0.17^{\circ} \mathrm{C}\right)$ temperatures were higher in HOT than COOL $(p<0.05)$ conditions. Shuttle running distance increased after both interventions $(p<0.001)$, with a non-significant $(p=0.131)$ but larger effect in HOT (315 $\mathrm{m}, d=1.18)$ than COOL $(207 \mathrm{~m}, d=0.51)$ conditions. Mean $(\sim 7 \%, p<0.001)$ and peak $(5 \%, p<0.05)$ power during repeatedsprinting increased following both interventions, whereas peak twitch force before the repeated-sprint assessment was $\sim 10 \%$ lower after the interventions $(p=0.001)$. Heart rate during the repeated-sprint warm-up was reduced $\left(\sim 6\right.$ beats. $\left.\mathrm{min}^{-1}\right)$ following both interventions $(p<0.01)$. Rectal temperature was $\sim 0.14^{\circ} \mathrm{C}$ lower throughout the repeatedsprint assessment after the interventions $(p<0.001)$, with larger effects in HOT than COOL during the warm-up ( $p=0.082 ; d=-0.53$ vs. $d=-0.15)$ and repeated-sprints $(p=0.081$; $d=-0.54$ vs. $d=-0.02)$. Skin temperature $(p=0.004, d=-1.11)$ and thermal sensation ( $p=0.015, d=-0.93$ ) were lower during the repeated-sprints after training in HOT than COOL. Sweat rate increased $\left(0.2{\left.\mathrm{~L} . \mathrm{h}^{-1}\right)}\right.$ ) only after training in HOT $(p=0.027 ; d=0.72)$. The intensive nature of brief repeated-sprint training induces similar improvements in repeated-sprint cycling ability in hot conditions and intermittent running performance in cool conditions, along with analogous physiological adaptations, irrespective of the environmental conditions in which training is undertaken.

Keywords: heat acclimation, thermoregulation, intermittent sports, running, repeated-sprint ability 


\section{INTRODUCTION}

Exercise-heat acclimation is used by endurance athletes to optimize performance when competing in the heat. The improvements in performance are attributed to enhanced sweating and skin blood flow responses, plasma volume expansion, increased cardiovascular stability, better fluid balance, and acquired thermal tolerance (Rowell, 1974; Sawka et al., 2011; Périard et al., 2015). The four main approaches conferring these adaptations include constant work rate exercise (e.g., $60 \%$ peak oxygen consumption: $\dot{\mathrm{V}}_{2 \text { peak }}$ ), self-paced exercise (i.e., variable work rate exercise), controlled hyperthermia (i.e., attain and maintain a target core temperature), and controlled heart rate (i.e., attain and maintain a target heart rate) heat acclimation (Daanen et al., 2018). The exercise associated with these approaches is typically endurance-based (i.e., low to moderate-intensity exercise; Nadel et al., 1974; Pandolf et al., 1977; Nielsen, 1998), with daily exposures of 60-90 min undertaken for 10-14 days (Nielsen et al., 1993; Lorenzo et al., 2010; Racinais et al., 2015).

The training required to optimize performance and heat tolerance in team-sport athletes, however, may differ to that commonly prescribed for endurance athletes. For sports such as rugby sevens, soccer, and field hockey, high-level performance involves the ability to repeat maximal or near-maximal sprint efforts (Girard et al., 2015). For example, a defining characteristic of international soccer players is their ability to perform $\sim 60 \%$ more sprinting than professional players of a lower standard (Mohr et al., 2003). When playing under heat stress, however, repeated-sprinting and jumping ability are compromised to a greater extent than when playing in temperate conditions (Mohr et al., 2010). This compromise occurs despite evidence that players adjust their physical activity patterns (e.g., decrease total and high-intensity running distance) in the heat to maintain the capacity to perform periodic sprint efforts when required at key moments in a match (i.e., pacing; Duffield et al., 2009; Mohr et al., 2012; Nassis et al., 2015). Repeated-sprint heat acclimation may thus be an approach that minimizes the impact of hot environmental conditions on repeated-sprint ability and low-to-moderate intensity exercise, whereby the pace and physical activity patterns adopted by team-sport athletes can be maintained throughout a match.

At the elite level, the training time and travel constraints of team-sport athletes are such that prolonged ( $>7$ days) interventions may be difficult to implement during the regular season (Chalmers et al., 2014). Short-term heat acclimation regimens involving repeated sprints may therefore be more appropriate, particularly since repeated-sprinting improves physical performance (i.e., repeated-sprint ability; Bishop et al., 2011), and short-term heat acclimation can initiate thermoregulatory adaptations and plasma volume expansion and improve perceptions of exertion and thermal comfort (Garrett et al., 2009, 2011; Chalmers et al., 2014). The distinction between repeated-sprint and intermittent or high-intensity interval training is important and lies with the intensity and length of the efforts, as well as the duration of recovery between efforts. Repeated sprinting is characterized by brief "all-out" efforts ( $\leq 10 \mathrm{~s})$ and incomplete recovery ( $\leq 60 \mathrm{~s}$ ), whereas intermittent sprinting involves longer recovery periods (60-300 s; Girard et al., 2011). High-intensity interval training involves short-to-long efforts (45-240 s) of high but not maximal intensity exercise, interspersed with recovery periods or varying length (e.g., work/rest ratio of $1: 1$ or more; Buchheit and Laursen, 2013).

Although repeated-sprint ability is regarded as a key performance determinant for team-sport success (Girard et al., 2011; Billaut et al., 2012; Sweeting et al., 2017), only one study appears to have used maximal (i.e., "all-out") repeated sprinting to promote heat adaptation over a short time frame ( $<7$ days). Petersen et al. (2010) had participants complete maximal cycling sprints on 4 consecutive days in $30^{\circ} \mathrm{C}$ and $61 \%$ relative humidity (RH) for a total heat exposure time of $150 \mathrm{~min}$. This heat training intervention decreased heart rate and thermal discomfort and improved thermal sensation at the end of a 30-min submaximal running test in the heat, relative to participants who trained in $20^{\circ} \mathrm{C}$ and $63 \% \mathrm{RH}$. However, short-term heat training did not influence repeated-sprint performance in temperate conditions $\left(24^{\circ} \mathrm{C}\right.$ and $\left.48 \% \mathrm{RH}\right)$. A more protracted maximal repeated-sprint protocol performed in $35^{\circ} \mathrm{C}$ and $60 \%$ $\mathrm{RH}$ conditions on either 8 consecutive days or every second day, yielded a similar adaptive response in thermoregulatory capacity and improvements in repeated-sprint performance, regardless of the approach (i.e., continuous or intermittent; Duvnjak-Zaknich et al., 2019). The improvements in performance were maintained for 2 weeks following completion of both the continuous and intermittent protocols, which provided a total exercise-heat exposure time of $324 \mathrm{~min}$ with a daily change in core temperature of $1.3-1.8^{\circ} \mathrm{C}$. In contrast, six sessions of Wingate interval training over 2 weeks in $40^{\circ} \mathrm{C}$ did not

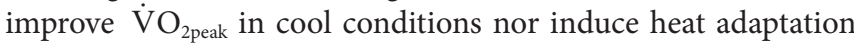
when assessed in $25^{\circ} \mathrm{C}$ conditions (Wingo et al., 2018). The authors concluded that the overall exposure time (165 min) and minimal increase in daily core temperature $\left(0.6-0.9^{\circ} \mathrm{C}\right)$ were insufficient to promote an adaptive response, as profuse sweating and elevated skin and core temperatures are required to induce adaptations (Sawka et al., 2011; Périard et al., 2015). Altogether, it appears that heat adaptations and performance improvements with short-to-medium-term maximal sprint heat acclimation protocols relate to both the magnitude and total duration of exercise-heat exposure, as well as the environmental conditions in which performance tests (e.g., aerobic capacity and repeated-sprint ability) are completed. The performance benefits may also be influenced by the fatiguing nature of the exercise and a lack of adequate recovery between training sessions and subsequent testing (Reeve et al., 2019).

The purpose of this study was to determine whether five sessions of repeated-sprint training over 7 days (2 days of recovery) in hot environmental conditions $\left(40^{\circ} \mathrm{C}\right.$ and $\left.40 \% \mathrm{RH}\right)$ induced adaptations commensurate with heat acclimation and greater improvements in repeated-sprint ability and aerobic performance than training in cool conditions $\left(20^{\circ} \mathrm{C}\right.$ and $40 \%$ $\mathrm{RH})$. To elucidate the potential pathway via which performance may be enhanced or influenced by cumulative muscle fatigue, a neuromuscular function assessment was also conducted. 
It was hypothesized that repeated-sprint training with $300 \mathrm{~min}$ of total heat exposure would initiate a heat acclimation response and enhance both repeated-sprint ability in the heat and aerobic performance in cool conditions.

\section{MATERIALS AND METHODS}

\section{Participants}

Twenty-five trained, non-heat acclimatized (June to November in Canberra, average high temperature of $15.5^{\circ} \mathrm{C}$ ), male teamsport athletes (regional level soccer, rugby, and Australian rules football) training $\sim 7 \mathrm{~h}$ per week participated in the study. Thirteen participants completed the training regimen in HOT conditions - age, body mass, height, and $\dot{\mathrm{VO}}_{2 \text { peak }}: 26 \pm 5$ year, $81.9 \pm 9.7 \mathrm{~kg}, 1.8 \pm 0.1 \mathrm{~m}$, and $50.7 \pm 3.9 \mathrm{ml} \cdot \mathrm{kg}^{-1} \cdot \mathrm{min}^{-1}$ (mean $\pm \mathrm{SD}$ ), respectively. Twelve participants completed the training regimen in COOL conditions - $23 \pm 4$ year, $81.7 \pm 10.5 \mathrm{~kg}, 1.8 \pm 0.1 \mathrm{~m}$, and $51.8 \pm 5.9 \mathrm{ml} \cdot \mathrm{kg}^{-1} \cdot \mathrm{min}^{-1}$. Participants were fully informed of the experimental procedures and potential risks prior to giving written informed consent. All participants completed an Adult Pre-exercise Screening Tool (Exercise \& Sport Science Australia, Ascot, Australia, 2011) before admission to the study. The protocol was approved by the University of Canberra Human Research Ethics Committee (17-115) and all procedures conformed to the standards of the Declaration of Helsinki.

\section{Experimental Design}

Participants visited the laboratory $\sim 1$ week prior to commencing the study for a familiarization session where the neuromuscular function assessment and repeated-sprint protocol were performed in cool conditions. Participants were then assigned via block randomization (Suresh, 2011) to either the HOT or COOL (i.e., control) training group in a counterbalanced order and undertook three pre-experimental tests, each separated by $48 \mathrm{~h}$ (Figure 1). The tests were repeated after the repeated-sprint training regimen and included a $\dot{\mathrm{V}} \mathrm{O}_{2 \text { peak }}$ test in cool conditions, a repeated-sprint test (RST) in the heat, which was immediately preceded and followed by a neuromuscular function assessment of the knee extensors in cool conditions, and a Yo-Yo Intermittent Recovery Test Level 1 (Yo-Yo IR1) conducted in cool conditions. After completing all pre-experimental tests, participants undertook a training regimen consisting of five sessions in 7 days in either the HOT or COOL condition. The regimen consisted of performing a repeated-sprint protocol on days 1 , 2, 4, 6, and 7 (Figure 1). Temperature and $\mathrm{RH}$ were standardized for all procedures undertaken in the HOT $\left(40^{\circ} \mathrm{C}\right.$ and $40 \%$ $\mathrm{RH})$ and COOL $\left(20^{\circ} \mathrm{C}\right.$ and $\left.40 \% \mathrm{RH}\right)$ conditions, except for the Yo-Yo IR1 test performed in $15-18^{\circ} \mathrm{C}$. All testing and training sessions were conducted at the same time of day for each individual.

\section{Testing and Training Procedures $\mathrm{vO}_{\text {2peak }}$ Test}

The incremental exercise test to determine $\dot{\mathrm{V}} \mathrm{O}_{2 \text { peak }}$ consisted of cycling on an electromagnetically braked cycle ergometer (Velotron, RacerMate Inc., Seattle, WA, USA) at an initial power output of $100 \mathrm{~W}$ with a $25 \mathrm{~W} \cdot \mathrm{min}^{-1}$ increase until volitional fatigue. Expired air was analyzed by paramagnetic $\mathrm{O}_{2}$ and infrared $\mathrm{CO}_{2}$ analyzers (ParvoMedics Inc., Salt Lake City, UT, USA). Heart rate was monitored continuously with a Polar transmitter-receiver (T-31 Polar Electro, Lake Success, USA) and captured within the Velotron software.

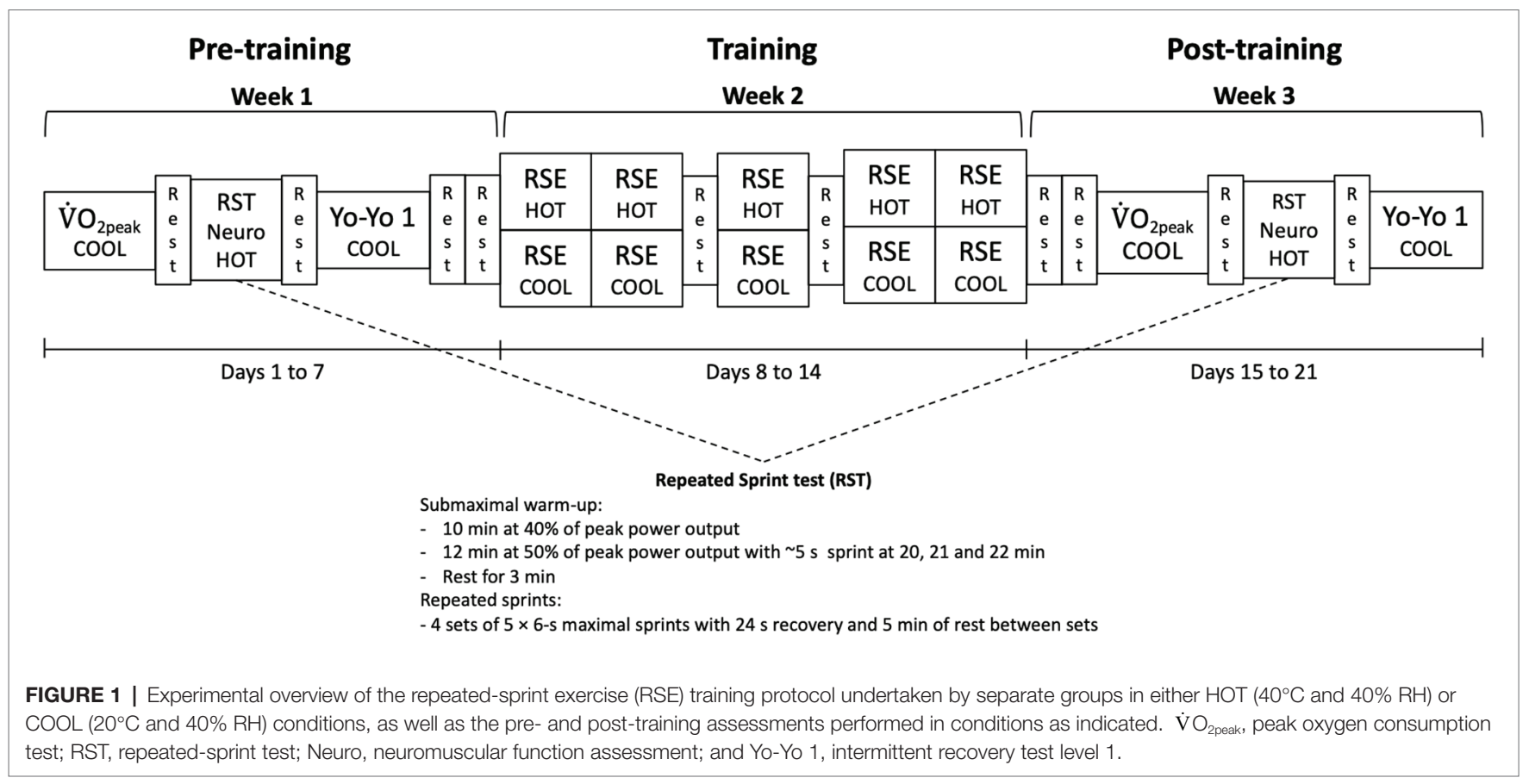




\section{Yo-Yo IR1 Test}

The Yo-Yo IR1 was used as a team-sport-specific field test, consisting of repeating $20-\mathrm{m}$ shuttle runs at increasing speeds, starting at $10 \mathrm{~km} \cdot \mathrm{h}^{-1}$. Each run is interspersed with an active recovery period of $10 \mathrm{~s}$, consisting of $5 \mathrm{~m}$ of jogging/walking (Krustrup et al., 2003). Verbal encouragement was provided to participants when nearing the end of the test, which was conducted indoors on a synthetic running track.

\section{Repeated-Sprint Test}

On arrival to the laboratory ( $\sim 60 \mathrm{~min}$ prior to testing), participants emptied their bladder and provided a urine sample for measurement of urine specific gravity (USG: PEN-Urine SG, Atago, Tokyo, Japan). If USG was $>1.020$, a $300 \mathrm{ml}$ bolus of water was consumed within $10 \mathrm{~min}$. Participants were then weighed before changing into sporting attire (shorts, socks, and shoes) and self-inserting a general-purpose thermistor probe (TM400, Covidien, Mansfield, MA, USA) $12 \mathrm{~cm}$ beyond the anal sphincter to measure rectal temperature $\left(T_{\mathrm{re}}\right)$. Participants then rested in the seated position in cool conditions while instrumented with a heart rate monitor and skin temperature sensors (iButton, Maxim Integrated Products, San Jose, CA, USA) to calculate mean skin temperature $\left(T_{\mathrm{sk}}\right.$; Ramanathan, 1964). Baseline measurements were taken after $5 \mathrm{~min}$ in the supine position prior to undertaking the neuromuscular function assessment. Participants then entered the climate chamber set to HOT conditions and mounted a cycle ergometer (Wattbike Pro, Nottingham, UK). After 2 min of seated rest, a $22 \mathrm{~min}$ warm-up was initiated, consisting of cycling at $40 \%$ of the peak power output achieved during the $\mathrm{VO}_{2 \text { peak }}$ test for $10 \mathrm{~min}$, and then $50 \%$ of peak power output for the following $12 \mathrm{~min}$. At 20, 21, and $22 \mathrm{~min}$, a brief ( $5 \mathrm{~s}$ ) maximal sprint was performed. Participants then rested for $3 \mathrm{~min}$.

The repeated-sprint protocol consisted of four sets of $5 \times 6$-s maximal (i.e., all-out and standing) cycling sprints separated by $24 \mathrm{~s}$ of passive recovery and $5 \mathrm{~min}$ of seated rest between sets. The sprints were conducted on the Wattbike ergometer at air and magnetic resistances of 10 and 3, respectively. A countdown to start and finish each sprint was provided, as well as strong verbal encouragement. Ratings of perceived exertion (RPE; Borg, 1982) and thermal sensation (American Society of Heating, Refrigeration and Air-Conditioning, 1966) were recorded at the end of each set. Constant airflow $\left(\sim 12.5 \mathrm{~km} . \mathrm{h}^{-1}\right)$ was provided by an electric fan aimed at the torso and head of the participants during the repeated-sprint protocol. Participants were permitted to drink water ad libitum during the repeated-sprint protocol. Change in body mass was calculated at the conclusion of the "Neuromuscular Function Assessment" to determine whole-body sweat production with corrections for fluid ingested, urine losses, and sweat trapped in clothing.

\section{Neuromuscular Function Assessment}

Following baseline measurements for the RST, a neuromuscular function assessment consisting of voluntary and electrically-evoked contractions was performed with participants seated upright on a custom-built adjustable chair with the hips and knees flexed at $90^{\circ}$. Restraining straps placed across the chest and hips secured the participant in the chair and prevented extraneous movement, while a dynamometer (CAPTELS, Saint-Mathieu-de-Treviers,
France) was attached 3-5 $\mathrm{cm}$ above the tip of the lateral malleoli. During all contractions, the force signal was amplified, sent through an $\mathrm{A} / \mathrm{D}$ board, and sampled at $2,000 \mathrm{~Hz}$ by commercially available hardware and software (Biopac Student Lab 4.1, BIOPAC Systems Inc., Santa Barbara, California, USA). A high-voltage stimulator (Digitimer DS7AH, Digitimer, Welwyn Garden City, UK) was used to deliver a square-wave stimulus of $0.2 \mathrm{~ms}$ duration with a maximal voltage of $400 \mathrm{~V}$. The femoral nerve was stimulated by placing a cathode $(5 \mathrm{~mm}$ diameter) in the inguinal crease and an anode $(5 \times 9 \mathrm{~cm}$ TENS Pads, TENS Machines, West End, Queensland, Australia) in the gluteal fold. During the familiarization session, an isometric recruitment curve using motor nerve stimulation was drawn on the relaxed quadriceps to individualize the stimulus intensity. The current was increased in $20 \mathrm{~mA}$ increments until a plateau occurred in maximal twitch amplitude. Supramaximal stimulation for the experimental trials was ensured by increasing the final intensity by $50 \%$ (HOT: $205 \pm 73$ and COOL: $227 \pm 27 \mathrm{~mA}$; mean $\pm \mathrm{SD})$. The neuromuscular assessment consisted of a brief (5 s) maximal voluntary isometric contraction (MVC) of the knee extensors, on which a paired stimulus (doublet at $100 \mathrm{~Hz}$ ) was superimposed. This contraction was followed by another paired stimulus (i.e., potentiated doublet) and a single pulse (i.e., twitch) on the relaxed muscle, each interspersed by 5 s. During all MVCs, the participants were instructed to reach maximal force as quickly as possible and maintain this level for the duration of the contraction. Participants were strongly encouraged with verbal reinforcement and a visual display of force production. Approximately $30 \mathrm{~s}$ after completing the final sprint of the RST, participants made their way to the neuromuscular function chair where they repeated the neuromuscular function assessment.

\section{Repeated-Sprint Training}

Each session of the repeated-sprint training regimen was conducted as outlined above, with one group training in HOT and the other in COOL conditions. The training sessions did not include a neuromuscular function assessment. However, a 10-min cool-down at $40 \%$ of $\dot{\mathrm{VO}}_{2 \text { peak }}$ power output was added to extend total trial duration of each session to $60 \mathrm{~min}$ (including the 25 min warm-up). Throughout testing and training, the participants were asked to maintain a normal diet, but avoid the consumption of alcohol for $24 \mathrm{~h}$ and caffeine for $8 \mathrm{~h}$ prior to testing or training. Participants were also instructed to avoid strenuous training outside of the study protocol, maintain their usual aerobic/endurance training sessions, and complete a daily training diary, which was verified upon arrival for training.

\section{Data Analysis}

Repeated-sprint performance was analyzed by calculating mean and peak power output across each set. The percent decrement score $\{\%=[1-($ sprint $1+2+3+4+5) /$ best sprint $\times$ number of sprints] $\times 100\}$ was calculated from the mean and peak power outputs recorded across each set (Girard et al., 2011). The neuromuscular function analysis was performed using the Biopac software. Voluntary activation was calculated with the interpolated twitch technique $\{\mathrm{VA}(\%)=[1-$ (superimposed 
doublet/resting potentiated doublet) $] \times 100\}$. Force production in the calculation of voluntary activation was recorded as the average force of the $250 \mathrm{~ms}$ period prior to motor nerve stimulation. Peak twitch force was assessed from the resting twitches evoked after the MVCs. The highest of the three brief MVCs was selected to assess voluntary force production and activation, whereas the mean of the three twitches was used to analyze peak twitch force.

\section{Statistical Analysis}

An a priori power analysis for sample size estimation was conducted for changes in resting core temperature following short-term heat acclimation based on two studies with a partial eta-squared of 0.27 (Moss et al., 2019) and 0.10 (Reeve et al., 2019). Power analyses were also performed for changes in repeated-sprint performance with a partial eta-squared of 0.32 (Duvnjak-Zaknich et al., 2019) and Yo-Yo IR2 performance with a partial eta-squared of 0.92 (Racinais et al., 2014). With an alpha of 0.05 and power of 0.80 , the estimated sample size needed for repeated measures of within and between group comparisons was 8 and 20 for resting core temperature, and 8 and 4 for repeated-sprinting and Yo-Yo IR2 performance $\left(\mathrm{G}^{\star}\right.$ Power 3.1.9.6). Given the nature of the project and the potential for abandonment, 13 participants (one subsequent abandonment) per group were recruited to ensure that the study was well powered. A mixed linear modeling procedure was used to estimate means (fixed effects) and within- and between-subject variations (random effects, modeled as variances). The fixed effects were the underlying thermal condition in which training occurred (HOT or COOL), the time at which testing was conducted relative to the intervention (pre- or post-intervention), and the time within a testing session. The random effects were between-subject variances in performance, perceptual, and thermal adaptations, whereas within-subject variances represented typical variation in adaptation over the five training sessions. Where significant effects were established, pairwise comparisons were identified using the Bonferroni post hoc analysis adjusted for multiple comparisons. The rest, warm-up, and sprint segments of the RST were analyzed separately as they corresponded to periods of rest, submaximal constant work rate exercise, and maximal exercise, respectively. Paired sample $t$-tests were conducted to compare the within-group treatment (i.e., pre-to-post training intervention) effects on markers of hydration status (i.e., baseline body mass and USG). Model parameters and effects are reported as mean with 95\% confidence interval (CI: lower and upper bound) unless otherwise indicated. Cohen's $d$ effect sizes were interpreted as small (0.2), medium (0.5), and large (0.8; Cohen, 1992). All statistical analyses were performed using SPSS Software (IMB SPSS Statistic Version 25).

\section{RESULTS}

\section{Training Data}

$T_{\text {re }}$ during the repeated-sprint and cool-down segments (35 min) of the training sessions averaged $38.03^{\circ} \mathrm{C}(37.95-38.20)$ in COOL and $38.20^{\circ} \mathrm{C}(38.13-38.67)$ in HOT $(p=0.002)$, whereas
$T_{\text {sk }}$ was $27.51^{\circ} \mathrm{C}(26.82-28.05)$ in $\mathrm{COOL}$ and $35.88^{\circ} \mathrm{C}(35.57-$ $36.55)$ in $\operatorname{HOT}(p<0.001)$. The greater overall thermal strain was accompanied by a higher heart rate during these segments in HOT [169 beats. min $\left.^{-1}(166-172)\right]$ compared with COOL [159 beats. min $\left.^{-1}(157-161) ; p<0.001\right]$, and greater thermal sensation in HOT $[6.3(6.2-6.4)]$ than COOL [4.3 (4.1-4.6); $p<0.001]$.

\section{$\mathrm{vO}_{\text {2peak }}$ Test}

$\mathrm{VO}_{2 \text { peak }}$ values were similar between groups [mean: $51.9 \mathrm{ml}$. $\mathrm{kg}^{-1} \cdot \mathrm{min}^{-1} \quad(49.3-54.6) ; \quad p=0.563$ ], with no significant improvement following the intervention $\left[1.2 \mathrm{ml} \cdot \mathrm{kg}^{-1} \cdot \mathrm{min}^{-1}\right.$ $(-0.3-2.6) ; p=0.115 ; d=0.24]$. Maximum heart rate during the $\dot{\mathrm{V}}{ }_{2 \text { peak }}$ test was similar between groups [mean: 188 beats. $\min ^{-1}(184-191) ; p=0.815$ ] and decreased by 3 beats. $\min ^{-1}$ (1-5) after the training intervention $(p=0.007 ; d=-0.31)$.

\section{Yo-Yo IR1 Test}

Distance covered in the Yo-Yo IR1 test was similar between groups prior to [HOT: 1,434 m (1,248-1,619); COOL: $1,380 \mathrm{~m}$ $(1,187-1,573)$ ] and following [HOT: $1,749 \mathrm{~m}(1,564-1,934)$; COOL: $1,587 \mathrm{~m}(1,394-1,780)]$ the intervention (Figure 2; $p=0.399)$. After the training intervention, a $261 \mathrm{~m}(189-333)$ increase in Yo-Yo IR1 distance occurred ( $p<0.001 ; d=0.78$ ). The improvement in distance was not significantly different between the HOT and COOL groups [109 $\mathrm{m}$ (-35-252); $p=0.131]$, although the HOT group increased by $315 \mathrm{~m}(216-414$; $d=1.18)$ and the COOL group by $207 \mathrm{~m}(103-309 ; d=0.51)$.

\section{Repeated-Sprint Test - Physiological and Perceptual Responses}

Resting heart rate was similar between the HOT [60 beats. $\left.\min ^{-1}(55-66)\right]$ and COOL [65 beats. $\left.\mathrm{min}^{-1}(60-70)\right]$ groups prior to the intervention $(p=0.229)$, decreasing by 7 beats. $\min ^{-1}(4-10)$ after the intervention $(p<0.001 ; d=-0.73$ ). During the warm-up segment of the RST, an increase in heart rate occurred as a function of time (Figure 3; $p<0.001$; $d=1.94)$, with mean heart rate 8 beats. min $^{-1}(6-11)$ lower in the HOT group $(p<0.001 ; d=-0.49)$ and 4 beats. min $^{-1}$ (1-6) lower in the COOL group $(p=0.006 ; d=-0.22)$ following the intervention. The magnitude of decrease in heart rate post-intervention was not significantly different between groups $(p=0.591 ; d=-0.15)$. Throughout the repeated-sprint segment of the RST, heart rate was 2 beats. $\min ^{-1}(1-4)$ lower after completing the training intervention $(p<0.001 ; d=-0.24)$.

Resting $T_{\mathrm{re}}$ was similar prior to $\left[37.1^{\circ} \mathrm{C}(37.0-37.2)\right]$ and following $\left[37.0^{\circ} \mathrm{C}(36.9-37.1)\right]$ the training intervention in both groups (Figure $3 ; p=0.081 ; d=-0.46$ ). An increase in $T_{\mathrm{re}}$ was observed throughout the warm-up segment of the RST $(p<0.001 ; d=1.36)$, with mean $T_{\text {re }}$ lower by $0.11^{\circ} \mathrm{C}(0.05-0.16)$ after the training intervention $(p<0.001 ; d=-0.33)$. Although the lower $T_{\text {re }}$ observed following the training intervention was not significantly different between groups, a larger standardized effect was observed in the HOT $\left(\sim 0.17^{\circ} \mathrm{C}\right)$ compared with COOL $\left(\sim 0.08^{\circ} \mathrm{C}\right)$ group at rest $(p=0.670 ; d=-0.64$ vs. 

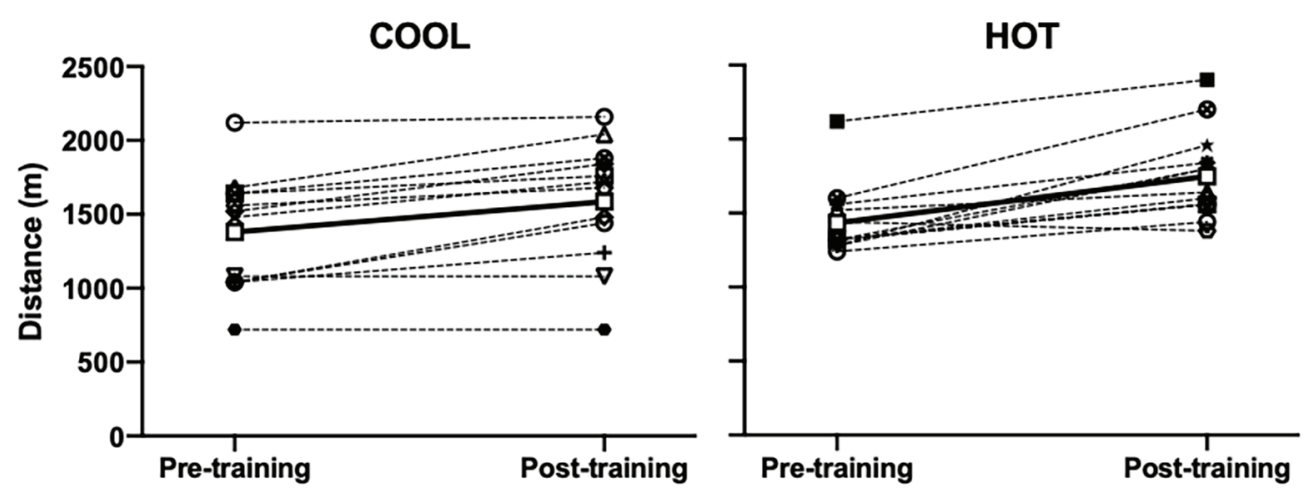

FIGURE 2 | Individual and mean Yo-Yo intermittent recovery test (level 1) distance covered prior to (pre-training) and following (post-training) a repeated-sprint training intervention undertaken in $\mathrm{COOL}\left(20^{\circ} \mathrm{C}\right.$ and $\left.40 \% \mathrm{RH}\right)$ or $\mathrm{HOT}\left(40^{\circ} \mathrm{C}\right.$ and $\left.40 \% \mathrm{RH}\right)$ conditions. Open squares and solid lines are means within each group. Symbols omitted for clarity. Post- vs. pre-intervention main effect $(p<0.001, d=0.78)$.

$d=-0.31$ ), during the warm-up ( $p=0.082 ; d=-0.53$ vs. $d=-0.15)$ and repeated-sprint $(p=0.081 ; d=-0.54$ vs. $d=-0.02$ ) segments.

Resting $T_{\text {sk }}$ was similar prior to $\left[30.5^{\circ} \mathrm{C}(30.0-30.9)\right]$ and following $\left[30.3^{\circ} \mathrm{C}(29.9-30.7)\right]$ the training intervention in both groups (Figure 3; $p=0.355$ ). During the warm-up segment of the RST, an increase in $T_{\text {sk }}$ was observed ( $\left.p<0.01 ; d=0.47\right)$, which following the training intervention was lower by $0.6^{\circ} \mathrm{C}$ $(0.4-0.7)$ in the HOT group $(p<0.001 ; d=-0.59)$ and higher by $0.2^{\circ} \mathrm{C}(0.0-0.4)$ in the COOL group ( $\left.p=0.015 ; d=0.09\right)$. There was a decrease in $T_{\text {sk }}$ during the repeated-sprint segment of the RST in both the HOT $(p<0.001 ; d=-1.03)$ and COOL $(p=0.010 ; d=-0.34)$ groups following the training intervention, with a $0.8^{\circ} \mathrm{C}(0.3-1.3)$ greater reduction in the HOT group ( $p=0.004 ; d=-1.11)$.

RPE increased throughout the warm-up segment of the RST in both groups $(p<0.001 ; d=1.87)$, with a decrease of 1.0 unit $(0.6-1.4)$ noted following the COOL intervention $(p<0.001 ; d=-0.55)$, but not the HOT intervention $(p=0.928$; $d=0.01$; Figure 4). There was also an increase in RPE during the repeated-sprint segment of the RST $(p<0.05 ; d=1.50)$, with values remaining similar between groups following the intervention $(p=0.904)$. Thermal sensation at rest was unaffected by either intervention ( $p=0.927)$. During the warm-up segment of the RST, an increase in thermal sensation was noted $(p<0.01$; $d=0.78$ ), which was lower by 0.5 units (0.1-0.8) following the training intervention in the HOT compared with COOL group ( $p=0.016 ; d=-0.81$ ). A similar response was observed during the repeated-sprint segment of the RST, where thermal sensation was lower by 0.4 units $(0.1-0.8)$ in the HOT compared with COOL group after the training intervention $(p=0.015$; $d=-0.93)$.

\section{Repeated-Sprint Test - Performance Responses}

Mean power across the RST was similar between groups $(p=0.547)$ but increased in both the COOL [48 W (27-68); $p<0.001 ; d=0.36$ ] and HOT [79 W (59-98); $p<0.001$; $d=0.52$ ] groups following the intervention (Figure 5).
The percent decrement score for mean power was significantly different between groups ( $p=0.030 ; d=0.68$ ). A significant decrease in the percent decrement score for mean power was observed following the intervention $[2.2 \%(0.3-4.1) ; p=0.025$; $d=-0.58]$. Although not significantly different between the HOT and COOL groups [2.7\% (-1.2-6.5); $p=0.167]$, the decrease in the percent decrement score was mostly due to the large $[3.9 \%(1.0-6.8)]$ reduction in the COOL $(d=-1.02)$ compared with HOT [1.2\% (-4.2-1.7); $d=-0.22]$ group. Peak power during the RST was similar between groups $(p=0.364)$ but increased following the intervention in the COOL [25 W (1.8-49); $p=0.035 ; d=0.17$ ] and HOT [67 W (44-89); $p<0.001 ; d=0.38$ ] groups. The percent decrement score for peak power was similar between groups $(p=0.445)$ and decreased after the intervention $(p=0.005 ; d=-0.67)$.

\section{Neuromuscular Function Assessment}

A significant decrease in MVC force was evident following the RST (Table 1; $p<0.001 ; d=-1.32$ ). Although force production capacity did not change after the training intervention ( $p=0.888 ; d=-0.01)$, a slightly lower force production was noted Pre-RST after the training intervention [921 (862-982) to $896 \mathrm{~N}$ (835-958); $d=-0.18]$. Voluntary activation was unaffected by the RST ( $p=0.289)$ or the intervention $(p=0.637)$. Peak twitch force decreased significantly Post-RST both prior to $(p<0.001 ; d=-2.72)$ and following $(p<0.001 ; d=-2.75)$ the interventions. Moreover, peak twitch force during the Pre-RST was lower after the training intervention $(p=0.001$; $d=-0.45)$.

\section{Hydration}

Baseline body mass prior to and following the training intervention was similar within the COOL [82.1 kg (78.3-85.9); $p=0.266]$ and HOT [81.6 kg (77.8-85.4); $p=0.967$ ] groups (values are means of pre- and post-intervention), as was USG [COOL: 1.014 (1.011-1.018); $p=0.435$ and HOT: 1.018 (1.015-1.021); $p=0.167]$. Sweat rate during the RST in the COOL group was unchanged following the training intervention [1.2 L.h L $\left.^{-1}(1.1-1.4) ; p=0.148 ; d=0.41\right]$, whereas an increase 

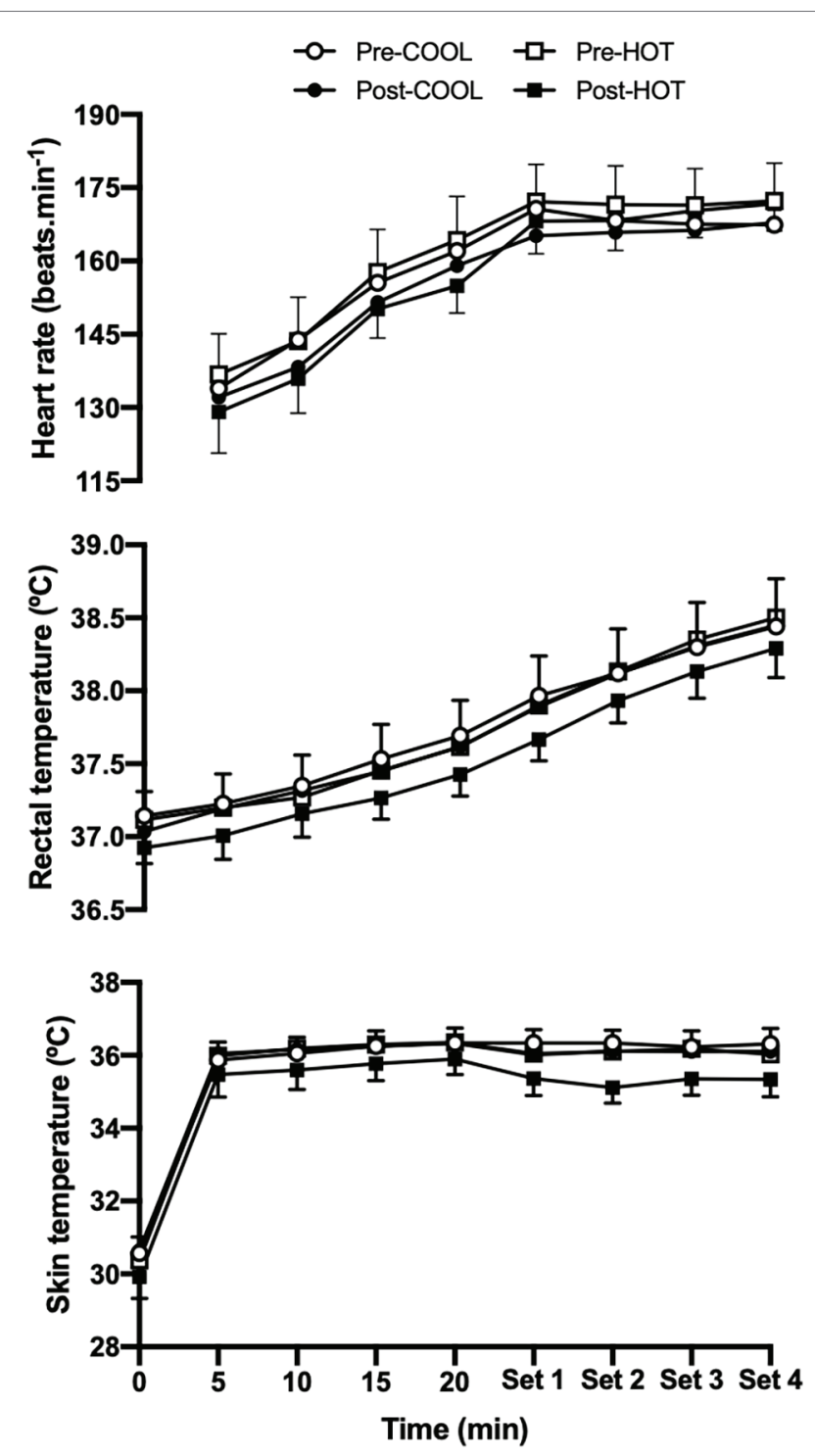

FIGURE 3 | Heart rate, rectal temperature, and mean skin temperature during a RST including a 22 min submaximal warm-up and four sets of repeated sprints performed in $40^{\circ} \mathrm{C}$ and $40 \% \mathrm{RH}$ prior to and following a repeated-sprint training intervention undertaken in $\mathrm{COOL}\left(20^{\circ} \mathrm{C}\right.$ and $\left.40 \% \mathrm{RH}\right)$ or HOT $\left(40^{\circ} \mathrm{C}\right.$ and $\left.40 \% \mathrm{RH}\right)$ conditions. Data are mean with $95 \% \mathrm{Cl}$. Symbols omitted for clarity. Heart rate: post- vs. pre-intervention main effect (warm-up: $p<0.001, d=-0.35$; sprints: $p<0.001, d=-0.24)$. Rectal temperature: post- vs. pre-intervention main effect (warm-up: $p<0.001, d=-0.33$; sprints: $p<0.001, d=-0.26$ ). Skin temperature: post- vs. pre-intervention within condition effect for HOT (warm-up: $p<0.001, d=-0.59$; sprints: $p<0.001, d=-1.03)$.

occurred in the HOT group from pre $\left[1.2{\mathrm{~L} . \mathrm{h}^{-1}}^{(0.8-1.5)]}\right.$ to post $\left[1.4\right.$ L.h $\left.^{-1}(1.0-1.8)\right]$ intervention $(p=0.027 ; d=0.72)$. Fluid intake during the RST remained unchanged by the training intervention in either the COOL [0.84 L (0.69-0.99); $p=0.447]$ or HOT [0.93 L (0.78-1.08); $p=0.413$ ] group. Percent body mass losses during the RST were similar in the COOL $[0.3 \%$ $(0.1-0.5) ; p=0.958]$ and HOT [0.1\% (-0.1-0.3); $p=0.065$; $d=-0.53]$ groups prior to and after the intervention.

\section{DISCUSSION}

The aim of this study was to examine the physiological adaptations and performance changes associated with short-term repeatedsprint training in $40^{\circ} \mathrm{C}$ (HOT) compared with $20^{\circ} \mathrm{C}$ (COOL) conditions in team-sport athletes. Our data indicate the emergence of heat acclimation in those having trained in the heat, with a decrease in $T_{\text {sk }}\left(\sim 0.7^{\circ} \mathrm{C}\right)$ and thermal sensation $(\sim 0.5)$, along with an increase in whole-body sweat rate $\left(0.2{\left.\mathrm{~L} . \mathrm{h}^{-1}\right)}^{-1}\right.$ during the post-intervention RST. Although not significantly different, slightly larger post-intervention improvements in $T_{\text {re }}$ were noted in the HOT relative to the COOL group. From a performance perspective, $\dot{\mathrm{VO}}_{2 \text { peak }}$ was unaffected by repeated-sprint training in either condition. In contrast, total running distance covered in the Yo-Yo IR1 test was increased in both groups $(\sim 18 \%)$, with a $\sim 50 \%$ larger, albeit non-significant, post-intervention improvement observed in the HOT group. The repeated-sprint training intervention in both HOT and COOL conditions also improved repeated-sprint cycling ability in the heat (mean power: $\sim 7 \%$, peak power: $\sim 5 \%$ ). These improvements in performance occurred despite the presence of skeletal muscle fatigue in both groups, as demonstrated by a $\sim 10 \%$ reduction in peak twitch force Pre-RST following the training intervention. These data indicate that five sessions of repeated-sprint training in HOT and COOL conditions over 7 days improved repeated-sprint ability in the heat and enhanced sport-specific endurance performance (i.e., 20-m shuttle run at increasing speeds) in cool conditions. From a physiological perspective, both training interventions induced an adaptive response, with partial heat adaptations emerging after training was conducted under heat stress. Taken together, these data indicate that the novel and intensive nature of a brief repeated-sprint training protocol induces analogous performance improvements and relatively similar physiological adaptations, irrespective of the environmental conditions in which it is undertaken.

\section{Adaptation to the Heat}

Investigations into the adaptive response of short-term heat acclimation regimens have increased in recent years, particularly for their use in team sports (Sunderland et al., 2008; Chalmers et al., 2014; Reeve et al., 2019) as pre-season training camps, for tapering before competition, and as in-season performanceenhancing camps (Périard et al., 2015). Chalmers et al. (2014) suggested that five 60 -min high-intensity sessions are required to improve aerobic performance in hot and potentially temperate environmental conditions in response to the development of cardiovascular, thermoregulatory, and metabolic adaptations. Data from previous short-to-medium term interval and repeatedsprint studies indicate that a sufficient thermal stimulus, both in terms of magnitude and duration, is required to promote the induction of heat acclimation (Petersen et al., 2010; Wingo et al., 2018; Duvnjak-Zaknich et al., 2019). For example, a 4-day program (total of $150 \mathrm{~min}$ ) of repeated-sprint training in $30^{\circ} \mathrm{C}$ led to a lower heart rate and thermal perception at the end of a 30-min run in the heat; however, it did not elicit changes in core and skin temperature or sweat rate (Petersen et al., 2010). Conversely, an 8-day continuous or 

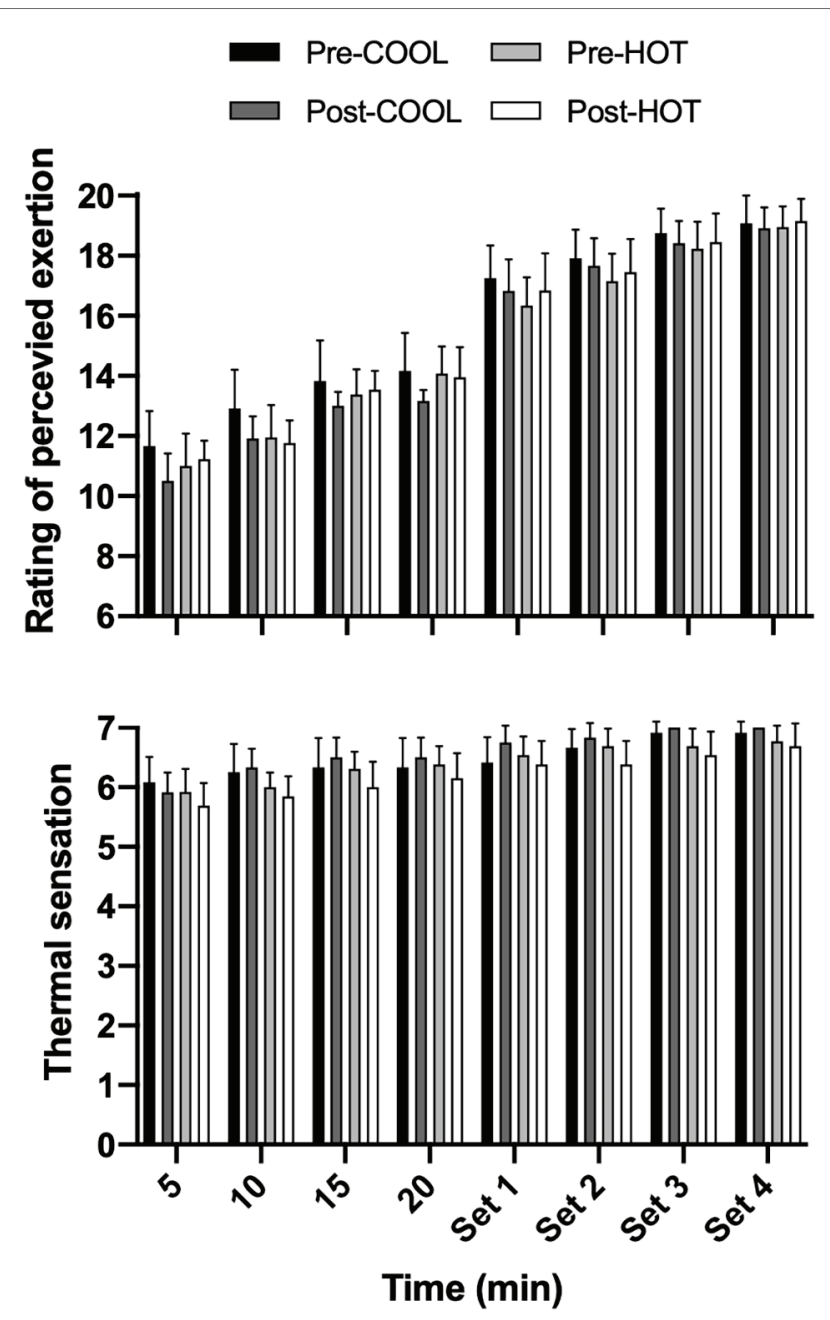

FIGURE 4 | Rating of perceived exertion and thermal sensation during a RST including a 22 min submaximal warm-up and four sets of repeated sprints performed in $40^{\circ} \mathrm{C}$ and $40 \% \mathrm{RH}$ prior to and following a repeated-sprint training intervention undertaken in $\mathrm{COOL}\left(20^{\circ} \mathrm{C}\right.$ and $\left.40 \% \mathrm{RH}\right)$ or $\mathrm{HOT}\left(40^{\circ} \mathrm{C}\right.$ and $40 \% \mathrm{RH}$ ) conditions. Data are mean with $95 \% \mathrm{Cl}$. Symbols omitted for clarity. Thermal sensation: post-intervention HOT and COOL between condition effects (warm-up: $p<0.016, d=-0.81$; sprints: $p<0.015, d=-0.93$ ).

intermittent repeated-sprint protocol in $35^{\circ} \mathrm{C}$ led to an increase in sweat rate and the maintenance of a similar core temperature while cycling at a higher power output (Duvnjak-Zaknich et al., 2019). Several studies utilizing short-term high-intensity intermittent exercise (i.e., submaximal intervals with longer recovery) have also demonstrated evidence of partial heat acclimation (e.g., lower heart rate, decreased core and skin temperatures, improved thermal comfort, and increased sweat rate; Brade et al., 2013; Kelly et al., 2016; Schmit et al., 2018; Reeve et al., 2019). In the current study, total exposure time during the five repeated-sprint training sessions over 7 days was $300 \mathrm{~min}$, with a daily increase in $T_{\text {re }}$ and $T_{\text {sk }}$ of $\sim 1.6$ and $6.5^{\circ} \mathrm{C}$, respectively, in the $\mathrm{HOT}$ group, relative to an increase in $T_{\text {re }}$ of $\sim 1.2^{\circ} \mathrm{C}$ and decrease in $T_{\text {sk }}$ of $\sim 3.0^{\circ} \mathrm{C}$ in those training in COOL conditions. The increase in heart rate was also more
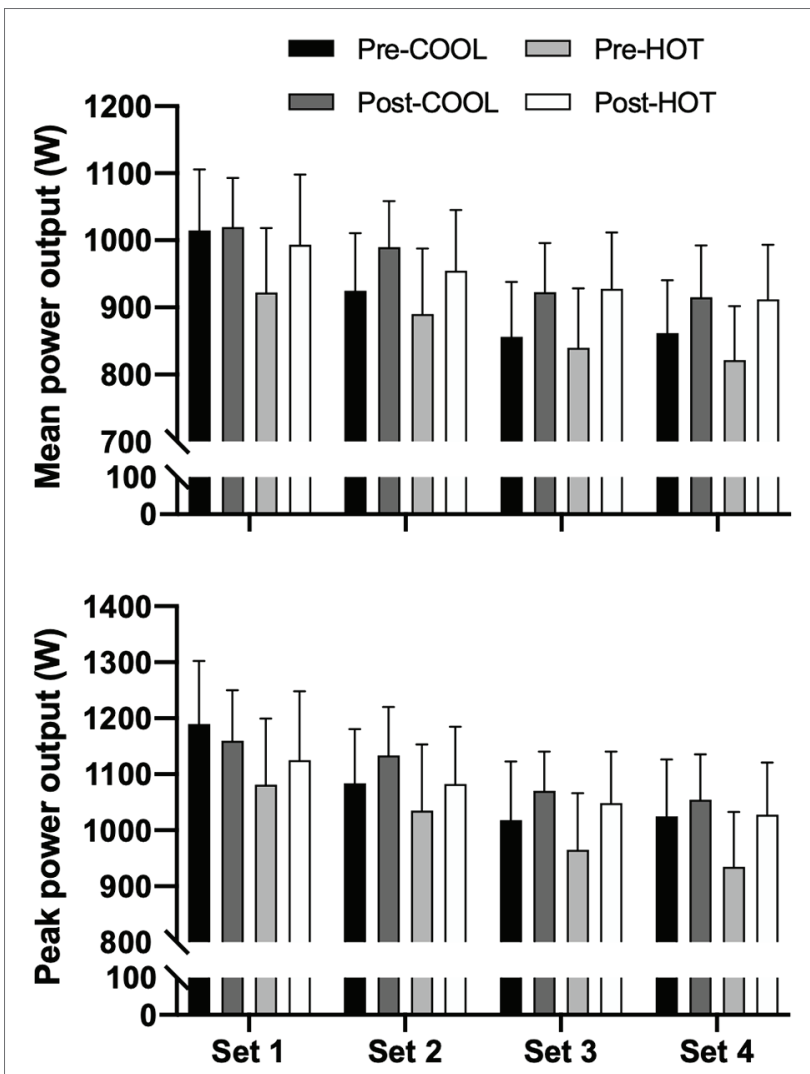

FIGURE 5 | Mean and peak power output across sets during a four-set RST performed in $40^{\circ} \mathrm{C}$ and $40 \% \mathrm{RH}$ prior to and following a repeated-sprint training protocol undertaken in $\mathrm{COOL}\left(20^{\circ} \mathrm{C}\right.$ and $\left.40 \% \mathrm{RH}\right)$ or $\mathrm{HOT}\left(40^{\circ} \mathrm{C}\right.$ and $\left.40 \% \mathrm{RH}\right)$ conditions. Data are mean with 95\% Cl. Symbols omitted for clarity. Mean power output: post- vs. pre-intervention main effect $(p<0.001, d=0.45)$. Peak power output: post- vs. pre-intervention main effect $(p<0.001, d=0.28)$.

pronounced in the HOT compared with COOL group during daily training, with $\sim 77$ vs. $70 \%$ and $\sim 90$ vs. $85 \%$ of maximum heart rate maintained during the warm-up and repeated-sprint segment of the RST, respectively. These responses indicate that a greater thermal stimulus for adaptation was provided to participants training in the heat.

In line with the provision of a greater thermal stimulus for adaption was an increase in sweat rate and reduction in $T_{\text {sk }}$ during the entire RST after training in the HOT condition. These adaptations were coupled with non-significant, but larger standardized effects for a lower $T_{\text {re }}\left(\sim 0.1^{\circ} \mathrm{C}\right)$ across all phases of the RST (i.e., rest, warm-up, and repeated sprints; Figure 3) in the HOT compared with COOL condition. However, given the relatively small differences noted between conditions, it is debatable whether the adaptations noted in the heat had an appreciable physiological impact, despite improving thermal perception (Figure 4). The similar adaptive response observed between groups highlights the partial heat acclimation phenotype exhibited by athletes regularly training in cool conditions (Avellini et al., 1982; Armstrong and Pandolf, 1988), which could partly explain the lack of a greater difference in thermoregulatory improvements (Ravanelli et al., 2020), with 
TABLE 1 | Maximal voluntary isometric contraction (MVC) force production, voluntary activation, and peak twitch force prior to (Pre) and following (Post) a repeated-sprint test (RST) in the heat.

\begin{tabular}{|c|c|c|c|c|c|c|}
\hline & & \multicolumn{2}{|c|}{ COOL } & \multicolumn{2}{|c|}{ НОТ } & \multirow{2}{*}{ Main effects } \\
\hline & & Pre-training & Post-training & Pre-training & Post-training & \\
\hline \multirow[t]{3}{*}{$\operatorname{MVC}(\mathrm{N})$} & Pre-RST & $924(682-1,167)$ & $911(618-1,205)$ & $920(645-1,195)$ & $878(583-1,172)$ & COOL vs. HOT: $p=0.669, d=0.15$ \\
\hline & & & & & & Post-RST vs. Pre-RST: $p<0.001, d=-1.32$ \\
\hline & Post-RST & 726 (515-938) & $772(506-1,038)$ & $693(424-962)$ & 688 (402-973) & Post-training vs. Pre-training: $p=0.888, d=-0.01$ \\
\hline \multirow{3}{*}{$\begin{array}{l}\text { Voluntary } \\
\text { activation (\%) }\end{array}$} & Pre-RST & $92(80-104)$ & $93(82-105)$ & 95 (86-105) & 95 (85-104) & COOL vs. HOT: $p=0.185, d=-0.34$ \\
\hline & & & & & & Post-RST vs. Pre-RST: $p=0.289, d=-0.32$ \\
\hline & Post-RST & $90(74-106)$ & $90(69-111)$ & $91(70-112)$ & $95(82-107)$ & Post-training vs. Pre-training: $p=0.637, d=0.10$ \\
\hline \multirow{3}{*}{$\begin{array}{l}\text { Peak twitch } \\
\text { force }(N)\end{array}$} & Pre-RST & 182 (103-261) & 167 (99-234) & 169 (104-234) & 151 (29-209) & COOL vs. HOT: $p=0.367, d=0.39$ \\
\hline & Post-RST & $80(28-132)$ & $95(66-124)$ & $79(32-126)$ & $75(32-118)$ & Post-RST vs. Pre-RST: $p<0.001, d=-2.76$ \\
\hline & & & & & & Post-training vs. Pre-training: $p=0.001, d=-0.19$ \\
\hline
\end{tabular}

The neuromuscular assessment was conducted before (Pre) and after (Post) a repeated-sprint training intervention undertaken in $\mathrm{COOL}\left[20^{\circ} \mathrm{C}\right.$ and $40 \%$ relative humidity ( $\mathrm{RH}$ )] or HOT $\left(40^{\circ} \mathrm{C}\right.$ and $\left.40 \% \mathrm{RH}\right)$ conditions. Data are mean (95\% confidence interval).

this trained cohort requiring a greater thermal stimulus for adaptation. Although the current results indicate the emergence of thermoregulatory and perceptual adaptations in the HOT group, there appears to be potential for an extended repeatedsprint training regimen to more fully induce heat acclimation in trained individuals. Moreover, limiting convective airflow when training, particularly during the recovery segments between sprint sets, would induce greater thermal strain by reducing evaporative heat loss and potentially promote a greater adaptive response.

\section{Performance Improvement}

The improvements in thermoregulatory capacity and cardiovascular stability afforded by heat acclimation are accompanied by attenuation of the deleterious impact of heat stress on aerobic performance (Périard et al., 2015; Tyler et al., 2016). In trained individuals, only five high-intensity sessions of $60 \mathrm{~min}$ may be required to improve aerobic-based performance in hot and cool conditions (Chalmers et al., 2014). However, there is disparity in the performance outcomes between studies utilizing this approach, which is inherently linked to the nature (i.e., repeated or intermittent sprint exercise) and brevity of the regimens (i.e., total duration of exposure), as well as the inter-individual variability in responsiveness. For example, five high-intensity interval training sessions over 9 days in $39^{\circ} \mathrm{C}$ decreased RPE and lactate concentration during submaximal exercise in the heat (Kelly et al., 2016). In well-trained females, a short-term high-intensity intermittent running regimen improved exercise capacity during the Loughborough Intermittent Shuttle Tests in $30^{\circ} \mathrm{C}$ heat by $33 \%$, while maximal $15 \mathrm{~m}$ sprint time was unaffected (Sunderland et al., 2008). A tendency for improved work capacity in the latter part of an intermittentsprint protocol under heat stress $\left(35^{\circ} \mathrm{C}\right)$ was reported following 5 days of heat acclimation performing high-intensity interval work (Brade et al., 2013). Conversely, short-term repeated-sprint training under heat stress $\left(30^{\circ} \mathrm{C}\right)$ did not influence repeatedsprint alibility in cool conditions $\left(24^{\circ} \mathrm{C}\right.$; Petersen et al., 2010).
In the current study, repeated-sprint training in both groups increased total distance in a Yo-Yo IR1 test performed in cool conditions, with a larger but non-significant effect noted in the HOT $(315 \mathrm{~m})$ compared with the COOL $(207 \mathrm{~m})$ group (Figure 2). Our data further indicate improved repeated-sprint ability in the heat in both groups, with higher mean and peak power outputs across the RST after the training interventions (Figure 5). A lower percent decrement score was also observed for mean and peak power output following the intervention in both the HOT and COOL groups. Taken together, the training intervention in both HOT and COOL conditions enhanced aerobic performance in cool conditions and repeatedsprint ability in the heat. Given the nature of the intervention (i.e., maximal sprinting), it appears that the novelty of maximal sprinting induces a similar performance enhancement, irrespective of the environmental conditions in which it is undertaken.

Interestingly, the improvement in repeated-sprint ability noted in the current study may have been dampened, as evidenced by a decrease in force production capacity (i.e., peak twitch force; Table 1) during the neuromuscular assessment Pre-RST following the intervention. This observation is suggestive of accumulated skeletal muscle fatigue due to a lack of adequate recovery between maximal exercise sessions, as a previous study demonstrated that passive heating for 11 consecutive days improved peak twitch force and MVC force production capacity in both normothermic and hyperthermic states (Racinais et al., 2017). In contrast, others have reported a failure to improve performance following short-term high-intensity training in the heat and ascribed the lack of improvement to the intense nature of such regimens. Reeve et al. (2019) demonstrated that $30 \mathrm{~min}$ of high-intensity interval training in the heat for 5 consecutive days reduced physiological and perceptual strain, but impaired endurance capacity during a heat stress test. It was suggested that such a regimen may not be ideal for athletes preparing to compete in the heat, as it may lead to a state of overreaching. A similar conclusion was drawn in a study where five consecutive high-intensity sessions in $30^{\circ} \mathrm{C}$ and $50 \% \mathrm{RH}$ appeared to induce a state of functional overreaching (Schmit et al., 2018). 
These studies, along with the current data, indicate that careful consideration and monitoring of athletes must be exercised when implementing a repeated-sprint heat acclimation regimen. This may be particularly relevant in team and racquet sports, as the performance benefits stemming from repeated high and maximal intensity bouts of exercise may, in the short term, be attenuated by the accumulation of fatigue.

\section{Summary}

A short-term repeated-sprint training intervention undertaken in HOT and COOL conditions improved Yo-Yo IR1 distance covered in cool conditions by $\sim 23$ and $16 \%$, respectively. Repeated-sprint ability in the heat was also improved in both groups, despite the presence of residual skeletal muscle fatigue, manifested as a reduction in peak twitch force prior to the RST conducted after the training intervention. Physiological adaptations commensurate with training (e.g., lower heart rate) were evident after five sessions of repeated sprinting in the HOT and COOL groups, with partial heat adaptations (i.e., increased sweat rate, lowered $T_{\text {sk }}$, and thermal sensation) emerging after training in the heat. These heat adaptations were negligible, however, as short-term repeated-sprint training improved repeated-sprint cycling ability in the heat, as well as high-intensity intermittent running performance in cool conditions, regardless of whether training was undertaken in HOT or COOL conditions.

\section{Perspective}

Preparing to compete in the heat might be difficult for highlevel team-sport athletes due to travel constraints and the time required to heat acclimate. Given that traditional heat acclimation approaches are endurance-based and require daily exposures of 60-90 min for 1-2 weeks, this study investigated whether a short-term repeated-sprint training intervention in $40^{\circ} \mathrm{C}$ induced adaptations commensurate with heat acclimation and greater improvements in repeated-sprint ability and aerobic performance, than training in $20^{\circ} \mathrm{C}$. It was observed that five sessions of repeated-sprint training in hot and cool conditions over 7 days improved repeated-sprint ability in the heat and enhanced 20-m shuttle running performance in cool conditions in team-sport athletes. Although training in the heat initiated the emergence of some heat adaptations, the novelty and intensive nature of the brief repeated-sprint training protocol induced similar performance and physiological improvements in the hot and cool conditions. The brevity of the protocol, however, also limited recovery time and resulted in skeletal

\section{REFERENCES}

Armstrong, L. E., and Pandolf, K. B. (1988). "Physical training, cardiorespiratory physical fitness and exercise-heat tolerance" in Human performance physiology and environmental medicine at terrestrial extremes. eds. K. B. Pandolf, M. N. Sawka and R. R. Gonzalez (Indianapolis, IN: Benchmark Press), 199-226.

American Society of Heating, Refrigeration and Air-Conditioning (1966). Thermal comfort conditions. ASHRAE standard 55:66. New York. muscle fatigue. As such, fatigue should be monitored during repeated-sprint training interventions to optimize recovery and performance benefits, with an extended protocol and additional recovery between sessions, potentially allowing for greater induction of heat acclimation in similarly trained individuals. The timeframe of implementation (e.g., pre-season training or pre-competition taper) for such an intervention should also be carefully considered.

\section{DATA AVAILABILITY STATEMENT}

The raw data supporting the conclusions of this article will be made available by the authors, without undue reservation.

\section{ETHICS STATEMENT}

The studies involving human participants were reviewed and approved by University of Canberra Human Research Ethics Committee. The patients/participants provided their written informed consent to participate in this study.

\section{AUTHOR CONTRIBUTIONS}

JP, OG, DP, and DB designed the study. JP and AW collected and analyzed the data. JP wrote the initial manuscript and all authors revised it and provided intellectual content. All authors contributed to the article and approved the submitted version.

\section{FUNDING}

This study was funded by the Australian Institute of Sport High Performance Sports Research Fund (PSRF20161712).

\section{ACKNOWLEDGMENTS}

The authors wish to thank Zoe Curran, Alec Mathias, Emily Thompson, Samantha Chin, and Cody Lindsay for their assistance in data collection. The authors also extend a special thank you to the participants who made this study possible. The results of the study are presented clearly, honestly, and without fabrication, falsification, or inappropriate data manipulation.

Avellini, B. A., Shapiro, Y., Fortney, S. M., Wenger, C. B., and Pandolf, K. B. (1982) Effects on heat tolerance of physical training in water and on land. J. Appl. Physiol. Respir. Environ. Exerc. Physiol. 53, 1291-1298. doi: 10.1152/jappl.1982.53.5.1291

Billaut, F., Gore, C. J., and Aughey, R. J. (2012). Enhancing team-sport athlete performance: is altitude training relevant? Sports Med. 42, 751-767. doi: 10.1007/BF03262293

Bishop, D., Girard, O., and Mendez-Villanueva, A. (2011). Repeated-sprint ability - part II: recommendations for training. Sports Med. 41, 741-756. doi: $10.2165 / 11590560-000000000-00000$ 
Borg, G. A. (1982). Psychophysical bases of perceived exertion. Med. Sci. Sports Exerc. 14, 377-381. doi: 10.1249/00005768-198205000-00012

Brade, C., Dawson, B., and Wallman, K. (2013). Effect of precooling and acclimation on repeat-sprint performance in heat. J. Sports Sci. 31, 779-786. doi: 10.1080/02640414.2012.750006

Buchheit, M., and Laursen, P. B. (2013). High-intensity interval training, solutions to the programming puzzle. Part II: anaerobic energy, neuromuscular load and practical applications. Sports Med. 43, 313-338. doi: 10.1007/s40279-013-0029-x

Chalmers, S., Esterman, A., Eston, R., Bowering, K. J., and Norton, K. (2014). Short-term heat acclimation training improves physical performance: a systematic review, and exploration of physiological adaptations and application for team sports. Sports Med. 44, 971-988. doi: 10.1007/s40279-014-0178-6

Cohen, J. (1992). A power primer. Psychol. Bull. 112, 155-159. doi: 10.1037/ 0033-2909.112.1.155

Daanen, H. A. M., Racinais, S., and Periard, J. D. (2018). Heat acclimation decay and re-induction: a systematic review and meta-analysis. Sports Med. 48, 409-430. doi: 10.1007/s40279-017-0808-x

Duffield, R., Coutts, A. J., and Quinn, J. (2009). Core temperature responses and match running performance during intermittent-sprint exercise competition in warm conditions. J. Strength Cond. Res. 23, 1238-1244. doi: 10.1519/ JSC.0b013e318194e0b1

Duvnjak-Zaknich, D. M., Wallman, K. E., Dawson, B. T., and Peeling, P. (2019). Continuous and intermittent heat acclimation and decay in team sport athletes. Eur. J. Sport Sci. 19, 295-304. doi: 10.1080/17461391.2018.1512653

Garrett, A. T., Goosens, N. G., Rehrer, N. J., Patterson, M. J., and Cotter, J. D. (2009). Induction and decay of short-term heat acclimation. Eur. J. Appl. Physiol. 107, 659-670. doi: 10.1007/s00421-009-1182-7

Garrett, A. T., Rehrer, N. J., and Patterson, M. J. (2011). Induction and decay of short-term heat acclimation in moderately and highly trained athletes. Sports Med. 41, 757-771. doi: 10.2165/11587320-000000000-00000

Girard, O., Brocherie, F., and Bishop, D. J. (2015). Sprint performance under heat stress: a review. Scand. J. Med. Sci. Sports 25, 79-89. doi: 10.1111/ sms. 12437

Girard, O., Mendez-Villanueva, A., and Bishop, D. (2011). Repeated-sprint ability - part I: factors contributing to fatigue. Sports Med. 41, 673-694. doi: 10.2165/11590550-000000000-00000

Kelly, M., Gastin, P. B., Dwyer, D. B., Sostaric, S., and Snow, R. J. (2016). Short duration heat acclimation in Australian football players. J. Sports Sci. Med. 15, 118-125.

Krustrup, P., Mohr, M., Amstrup, T., Rysgaard, T., Johansen, J., Steensberg, A., et al. (2003). The Yo-Yo intermittent recovery test: physiological response, reliability, and validity. Med. Sci. Sports Exerc. 35, 697-705. doi: 10.1249/01. MSS.0000058441.94520.32

Lorenzo, S., Halliwill, J. R., Sawka, M. N., and Minson, C. T. (2010). Heat acclimation improves exercise performance. J. Appl. Physiol. 109, 1140-1147. doi: 10.1152/japplphysiol.00495.2010

Mohr, M., Krustrup, P., and Bangsbo, J. (2003). Match performance of highstandard soccer players with special reference to development of fatigue. J. Sports Sci. 21, 519-528. doi: 10.1080/0264041031000071182

Mohr, M., Mujika, I., Santisteban, J., Randers, M. B., Bischoff, R., Solano, R., et al. (2010). Examination of fatigue development in elite soccer in a hot environment: a multi-experimental approach. Scand. J. Med. Sci. Sports 20(Suppl. 3), 125-132. doi: 10.1111/j.1600-0838.2010.01217.x

Mohr, M., Nybo, L., Grantham, J., and Racinais, S. (2012). Physiological responses and physical performance during football in the heat. PLoS One 7:e39202. doi: 10.1371/journal.pone.0039202

Moss, J. N., Bayne, F. M., Castelli, F., Naughton, M. R., Reeve, T. C., Trangmar, S. J., et al. (2019). Short-term isothermic heat acclimation elicits beneficial adaptations but medium-term elicits a more complete adaptation. Eur. J. Appl. Physiol. 120, 243-254. doi: 10.1007/s00421-019-04269-5

Nadel, E. R., Pandolf, K. B., Roberts, M. F., and Stolwijk, J. A. (1974). Mechanisms of thermal acclimation to exercise and heat. J. Appl. Physiol. 37, 515-520. doi: 10.1152/jappl.1974.37.4.515

Nassis, G. P., Brito, J., Dvorak, J., Chalabi, H., and Racinais, S. (2015). The association of environmental heat stress with performance: analysis of the 2014 FIFA World Cup Brazil. Br. J. Sports Med. 49, 609-613. doi: 10.1136/ bjsports-2014-094449

Nielsen, B. (1998). Heat acclimation - mechanisms of adaptation to exercise in the heat. Int. J. Sports Med. 19, S154-S156. doi: 10.1055/s-2007-971984
Nielsen, B., Hales, J. R., Strange, S., Christensen, N. J., Warberg, J., and Saltin, B. (1993). Human circulatory and thermoregulatory adaptations with heat acclimation and exercise in a hot, dry environment. J. Physiol. 460, 467-485. doi: 10.1113/jphysiol.1993.sp019482

Pandolf, K. B., Burse, R. L., and Goldman, R. F. (1977). Role of physical fitness in heat acclimatisation, decay and reinduction. Ergonomics 20, 399-408. doi: 10.1080/00140137708931642

Périard, J. D., Racinais, S., and Sawka, M. N. (2015). Adaptations and mechanisms of human heat acclimation: applications for competitive athletes and sports. Scand. J. Med. Sci. Sports 25, 20-38. doi: 10.1111/sms.12408

Petersen, C. J., Portus, M. R., Pyne, D. B., Dawson, B. T., Cramer, M. N., and Kellett, A. D. (2010). Partial heat acclimation in cricketers using a 4-day high intensity cycling protocol. Int. J. Sports Physiol. Perform. 5, 535-545. doi: 10.1123 /ijspp.5.4.535

Racinais, S., Buchheit, M., Bilsborough, J., Bourdon, P. C., Cordy, J., and Coutts, A. J. (2014). Physiological and performance responses to a trainingcamp in the heat in professional australian football players. Int. J. Sports Physiol. Perform. 9, 598-603. doi: 10.1123/ijspp.2013-0284

Racinais, S., Périard, J. D., Karlsen, A., and Nybo, L. (2015). Effect of heat and heat-acclimatization on cycling time-trial performance and pacing. Med. Sci. Sports Exerc. 47, 601-606. doi: 10.1249/MSS.0000000000000428

Racinais, S., Wilson, M. G., and Périard, J. D. (2017). Passive heat acclimation improves skeletal muscle contractility in humans. Am. J. Physiol. Regul. Integr. Comp. Physiol. 312, R101-R107. doi: 10.1152/ajpregu.00431.2016

Ramanathan, N. L. (1964). A new weighting system for mean surface temperature of the human body. J. Appl. Physiol. 19, 531-533. doi: 10.1152/jappl.1964.19.3.531

Ravanelli, N., Gagnon, D., Imbeault, P., and Jay, O. (2020). A retrospective analysis to determine if exercise training-induced thermoregulatory adaptations are mediated by increased fitness or heat acclimation. Exp. Physiol. doi: 10.1113/EP088385 [Epub ahead of print]

Reeve, T., Gordon, R., Laursen, P. B., Lee, J. K. W., and Tyler, C. J. (2019). Impairment of cycling capacity in the heat in well-trained endurance athletes after high-intensity short-term heat acclimation. Int. J. Sports Physiol. Perform. 14, 1058-1065. doi: 10.1123/ijspp.2018-0537

Rowell, L. B. (1974). Human cardiovascular adjustments to exercise and thermal stress. Physiol. Rev. 54, 75-159. doi: 10.1152/physrev.1974.54.1.75

Sawka, M. N., Leon, L. R., Montain, S. J., and Sonna, L. A. (2011). Integrated physiological mechanisms of exercise performance, adaptation, and maladaptation to heat stress. Compr. Physiol. 1, 1883-1928. doi: 10.1002/cphy.c100082

Schmit, C., Duffield, R., Hausswirth, C., Brisswalter, J., and Le Meur, Y. (2018). Optimizing heat acclimation for endurance athletes: high- versus low-intensity training. Int. J. Sports Physiol. Perform. 13, 816-823. doi: 10.1123/ijspp.2017-0007

Sunderland, C., Morris, J. G., and Nevill, M. E. (2008). A heat acclimation protocol for team sports. Br. J. Sports Med. 42, 327-333. doi: 10.1136/ bjsm.2007.034207

Suresh, K. (2011). An overview of randomization techniques: an unbiased assessment of outcome in clinical research. J. Hum. Reprod. Sci. 4, 8-11. doi: 10.4103/0974-1208.82352

Sweeting, A. J., Cormack, S. J., Morgan, S., and Aughey, R. J. (2017). When is a sprint a sprint? A review of the analysis of team-sport athlete activity profile. Front. Physiol. 8:432. doi: 10.3389/fphys.2017.00432

Tyler, C. J., Reeve, T., Hodges, G. J., and Cheung, S. S. (2016). The effects of heat adaptation on physiology, perception and exercise performance in the heat: a meta-analysis. Sports Med. 46, 1699-1724. doi: 10.1007/s40279-016-0538-5

Wingo, J. E., Katica, C. P., Nepocatych, S., Del Pozzi, A. T., and Ryan, G. A. (2018). Thermoregulatory adaptations following sprint interval training J. Hum. Perform. Extreme Environ. 14:8. doi: 10.7771/2327-2937.1106

Conflict of Interest: The authors declare that the research was conducted in the absence of any commercial or financial relationships that could be construed as a potential conflict of interest.

Copyright (c) 2020 Périard, Pyne, Bishop, Wallett and Girard. This is an openaccess article distributed under the terms of the Creative Commons Attribution License (CC BY). The use, distribution or reproduction in other forums is permitted, provided the original author(s) and the copyright owner(s) are credited and that the original publication in this journal is cited, in accordance with accepted academic practice. No use, distribution or reproduction is permitted which does not comply with these terms. 